\title{
A computational study on structure, stability and bonding in Noble Gas bound metal Nitrates, Sulfates and Carbonates (Metal $=\mathrm{Cu}, \mathrm{Ag}, \mathrm{Au}$ )
}

\author{
MANAS GHARA $^{\mathrm{a}}$, SUDIP PAN ${ }^{\mathrm{a}}$, JYOTIRMOY DEB $^{\mathrm{b}}$, ANAND KUMAR $^{\mathrm{a}, \mathrm{c}}$, \\ UTPAL SARKAR $^{\mathrm{b}, *}$ and PRATIM KUMAR CHATTARAJ ${ }^{\mathrm{a}, *}$ \\ ${ }^{a}$ Department of Chemistry and Centre for Theoretical Studies, Indian Institute of Technology, Kharagpur, \\ West Bengal 721 302, India \\ ${ }^{b}$ Department of Physics, Assam University, Silchar, Assam 788 011, India \\ ${ }^{c}$ Department of Chemistry, Indian Institute of Science Education and Research, Pune, \\ Maharashtra 411 008, India \\ e-mail: utpalchemiitkgp@yahoo.com; pkc@chem.iitkgp.ernet.in
}

MS received 14 March 2016; revised 2 June 2016; accepted 27 June 2016

\begin{abstract}
A density functional theory based study is performed to investigate the noble gas $(\mathrm{Ng}=\mathrm{Ar}-$ $\mathrm{Rn}$ ) binding ability of nitrates, sulfates and carbonates of noble metal (M). Their ability to bind $\mathrm{Ng}$ atoms is assessed through bond dissociation energy and thermochemical parameters like dissociation enthalpy and dissociation free energy change corresponding to the dissociation of $\mathrm{Ng}$ bound compound producing $\mathrm{Ng}$ and the respective salt. The zero-point energy corrected dissociation energy values per $\mathrm{Ng}$ atom for the dissociation process producing $\mathrm{Ng}$ atom(s) and the corresponding salts range within $6.0-13.1 \mathrm{kcal} / \mathrm{mol}$ in $\mathrm{NgCuNO}_{3}, 3.1-9.8 \mathrm{kcal} / \mathrm{mol}$ in $\mathrm{NgAgNO}_{3}, 6.0-13.2 \mathrm{kcal} / \mathrm{mol}$ in $\mathrm{NgCuSO}_{4}, 3.2-10.1 \mathrm{kcal} / \mathrm{mol}$ in $\mathrm{NgAgSO}_{4}$, $5.1-11.7 \mathrm{kcal} / \mathrm{mol}$ in $\mathrm{Ng}_{2} \mathrm{Cu}_{2} \mathrm{SO}_{4}, 2.5-8.6 \mathrm{kcal} / \mathrm{mol}$ in $\mathrm{Ng}_{2} \mathrm{Ag}_{2} \mathrm{SO}_{4}, 8.1-19.9 \mathrm{kcal} / \mathrm{mol}$ in $\mathrm{Ng}_{2} \mathrm{Au}_{2} \mathrm{SO}_{4}, 5.7-$ $12.4 \mathrm{kcal} / \mathrm{mol}$ in $\mathrm{NgCuCO}_{3}, 2.3-8.0 \mathrm{kcal} / \mathrm{mol}$ in $\mathrm{Ng}_{2} \mathrm{Ag}_{2} \mathrm{CO}_{3}$ and $7.3-18.2 \mathrm{kcal} / \mathrm{mol}$ in $\mathrm{Ng}_{2} \mathrm{Au}_{2} \mathrm{CO}_{3}$, with a gradual increase in moving from Ar to $\mathrm{Rn}$. For a given type of system, the stability of $\mathrm{Ng}$ bound analogues follows the order as $\mathrm{Au}>\mathrm{Cu}>\mathrm{Ag}$. All dissociation processes are endothermic in nature whereas they become endergonic as well in most of the cases of Kr-Rn bound analogues at $298 \mathrm{~K}$. Natural population analysis along with the computation of Wiberg bond indices, and electron density analyses provide insights into the nature of the Ng-M bonds. The Ng-M bonds can be represented as partial covalent bonds as supported by the different electron density descriptors.
\end{abstract}

Keywords. Noble metal-noble gas bond; dissociation energy; natural population analysis; electron density analysis.

\section{Introduction}

The synthesis of $\mathrm{Xe}^{+}\left[\mathrm{PtF}_{6}\right]^{-}$by Bartlett ${ }^{1}$ in 1962 induced immense interest into the chemistry of noble gas $(\mathrm{Ng})$ compounds and consequently enormous efforts were made by scientists both theoretically as well as experimentally during the last few decades. Ngs are generally known as chemically inactive elements and do not easily participate in chemical bonding. The low chemical tendency of Ngs to bind with other atoms makes it a challenge to discover new compounds involving Ngs. However, Bartlett's synthesis showed that the chemical bonding between Ngs and others atoms is definitely possible to produce new compounds but under certain specific physiochemical conditions and proper choice of reacting agents. To build up this

*For correspondence

Celebrating 100 years of Lewis Chemical Bond
$\mathrm{Ng}$ based field, scientists have been trying to develop a proper theory to get insight into the stability and chemical bonding of the new stable $\mathrm{Ng}$ compounds ${ }^{2-10}$ and also to synthesize these compounds using this theoretical understanding or vice versa. ${ }^{11-17}$ These efforts introduced a new dimension in the field of $\mathrm{Ng}$ compounds and showed that $\mathrm{Ngs}$ are not completely inactive.

Because of less reactivity, combining a coinage metal, also known as noble metal, and Ngs through chemical bond is also turned out to be a tough job. The effort in this regard was first made by Pyykkö ${ }^{18 a}$ by exploring the stability of $\mathrm{Ng}_{n} \mathrm{Au}^{+}(\mathrm{n}=1,2)$ through an in silico study which were later detected in mass spectrometry. ${ }^{18 \mathrm{~b}}$ Thereafter, $\mathrm{Ar}$ and $\mathrm{Kr}$ bound $\mathrm{AuCl}$ compounds were detected in microwave spectroscopy by Gerry et al., ${ }^{16 a}$ and $\mathrm{AuXe}_{4}^{2+}$ was synthesized as a reddish solid compound by Seidel et al. ${ }^{19}$ in the same year of 2000. Several $\operatorname{NgMX}(\mathrm{Ng}=\mathrm{Ar}, \mathrm{Kr}, \mathrm{Xe} ; \mathrm{M}=\mathrm{Cu}, \mathrm{Ag}, \mathrm{Au} ; \mathrm{X}=\mathrm{F}, \mathrm{Cl}$, $\mathrm{Br}$ ) compounds ${ }^{16}$ were then reported in subsequent years. 
Very recently, $\mathrm{NeAuF}$ was also reported by Wang and coworkers. ${ }^{20}$ Computational studies were devoted in order to analyze the stability of $\mathrm{NgMX}(\mathrm{X}=\mathrm{OH}, \mathrm{CN}$; $\mathrm{M}=\mathrm{Cu}, \mathrm{Ag}, \mathrm{Au}$ ) compounds. ${ }^{21,22}$ The possibility of $\mathrm{Ng}$ insertion within $\mathrm{M}-\mathrm{X}$ bonds in $\mathrm{MX}(\mathrm{X}=\mathrm{F}, \mathrm{OH} ; \mathrm{M}=$ $\mathrm{Cu}, \mathrm{Ag}, \mathrm{Au}$ ) compounds ${ }^{23}$ was also tested.

In the present work, we have investigated the interaction of $\mathrm{Ngs}$ with various nitrates, sulphates and carbonates of noble metals. Their efficacy to bind $\mathrm{Ngs}$ is analyzed through the computations of the dissociation energy and change in free energy $(\Delta \mathrm{G})$ and enthalpy $(\Delta \mathrm{H})$ at $298 \mathrm{~K}$ temperature, for the dissociations of $\mathrm{NgMNO}_{3}(\mathrm{M}=\mathrm{Cu}, \mathrm{Ag}), \mathrm{NgCu}\left(\mathrm{NO}_{3}\right)_{2}, \mathrm{NgMSO}_{4}(\mathrm{M}=$ $\mathrm{Cu}, \mathrm{Ag}), \mathrm{Ng}_{2} \mathrm{M}_{2} \mathrm{SO}_{4}(\mathrm{M}=\mathrm{Cu}, \mathrm{Ag}, \mathrm{Au}), \mathrm{NgCuCO}_{3}$ and $\mathrm{Ng}_{2} \mathrm{M}_{2} \mathrm{CO}_{3}(\mathrm{M}=\mathrm{Ag}, \mathrm{Au})$ into $\mathrm{Ng}(\mathrm{s})$ and the corresponding salts. The nature of bonding for the present cases

Color Code:

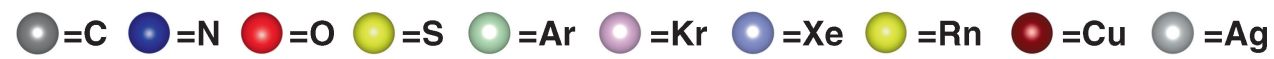
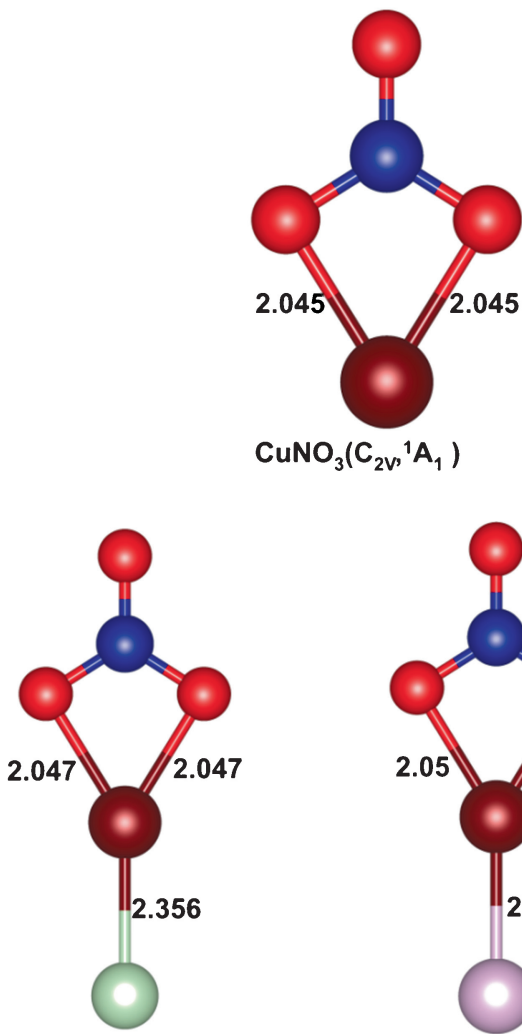

$\operatorname{ArCuNO}_{3}\left(C_{2 v},{ }^{1} A_{1}\right)$

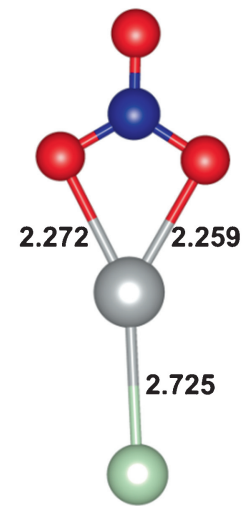

$\operatorname{ArAgNO}_{3}\left(C_{s},{ }^{1} \mathrm{~A}\right)$

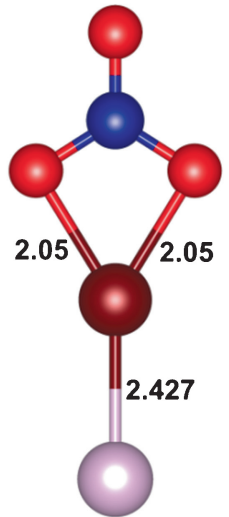

$\mathrm{KrCuNO}_{3}\left(C_{2 v},{ }^{1} A_{1}\right)$

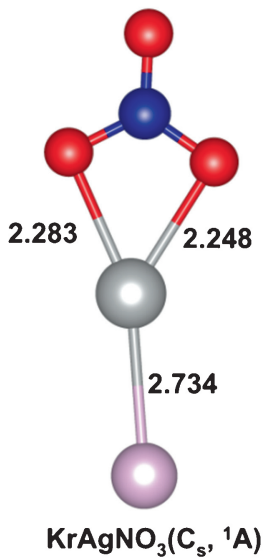

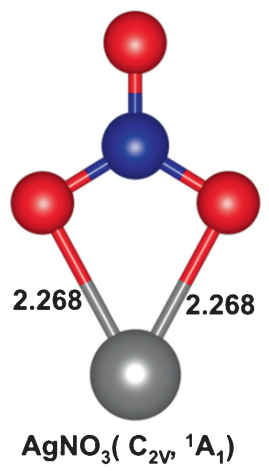
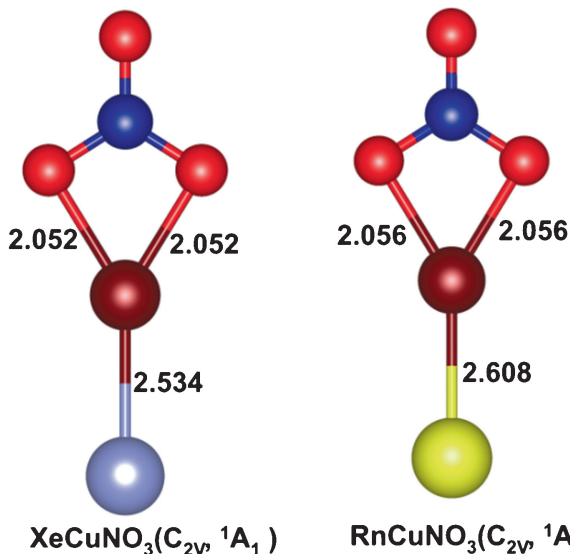

$\mathrm{RnCuNO}_{3}\left(\mathrm{C}_{2 \mathrm{v}},{ }^{1} \mathrm{~A}_{1}\right)$

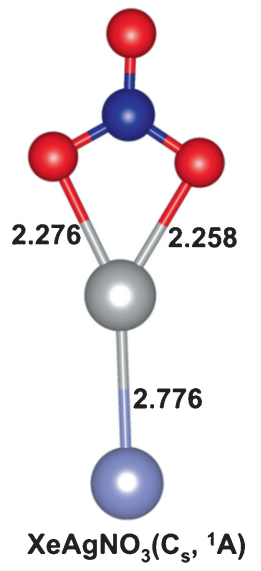

Figure 1. The optimized geometries of $\mathrm{MNO}_{3}$ and their $\mathrm{Ng}$ bound analogues $(\mathrm{M}=\mathrm{Cu}$, $\mathrm{Ag} ; \mathrm{Ng}=\mathrm{Ar}-\mathrm{Rn}$ ) at the MPW1B95/def2-TZVP level. Point groups along with their electronic states are given in the parentheses. The $\mathrm{Ng}-\mathrm{M}$ and $\mathrm{M}-\mathrm{O}$ bond distances are given in $\AA$ unit. 
is investigated through the natural population analysis (NPA), computation of Wiberg bond index (WBI) and a detailed analysis of the electron density. ${ }^{24}$

\section{Computational details}

All the studied compounds are fully optimized at the MPW1B95/def2-TZVP level of theory. The MPW1B95 functional is considered for the present study because a recent benchmarking study showed that this functional could provide the bond dissociation energy and bond distance very close to those obtained at the $\operatorname{CCSD}(\mathrm{T})$ level. ${ }^{25}$ An effective core potential $(\mathrm{ECP})^{26}$ is employed for the core electrons of $\mathrm{Ag}, \mathrm{Au}, \mathrm{Xe}$ and $\mathrm{Rn}$ atoms for the computational efficiency as well as to take care of the relativistic effect. Frequency calculations are also performed at the same level for the characterization of the nature of the stationary points, and to determine zeropoint energies (ZPE), enthalpies and free energies. It shows that there is no imaginary frequency for the sta-

\section{Colo Code:}
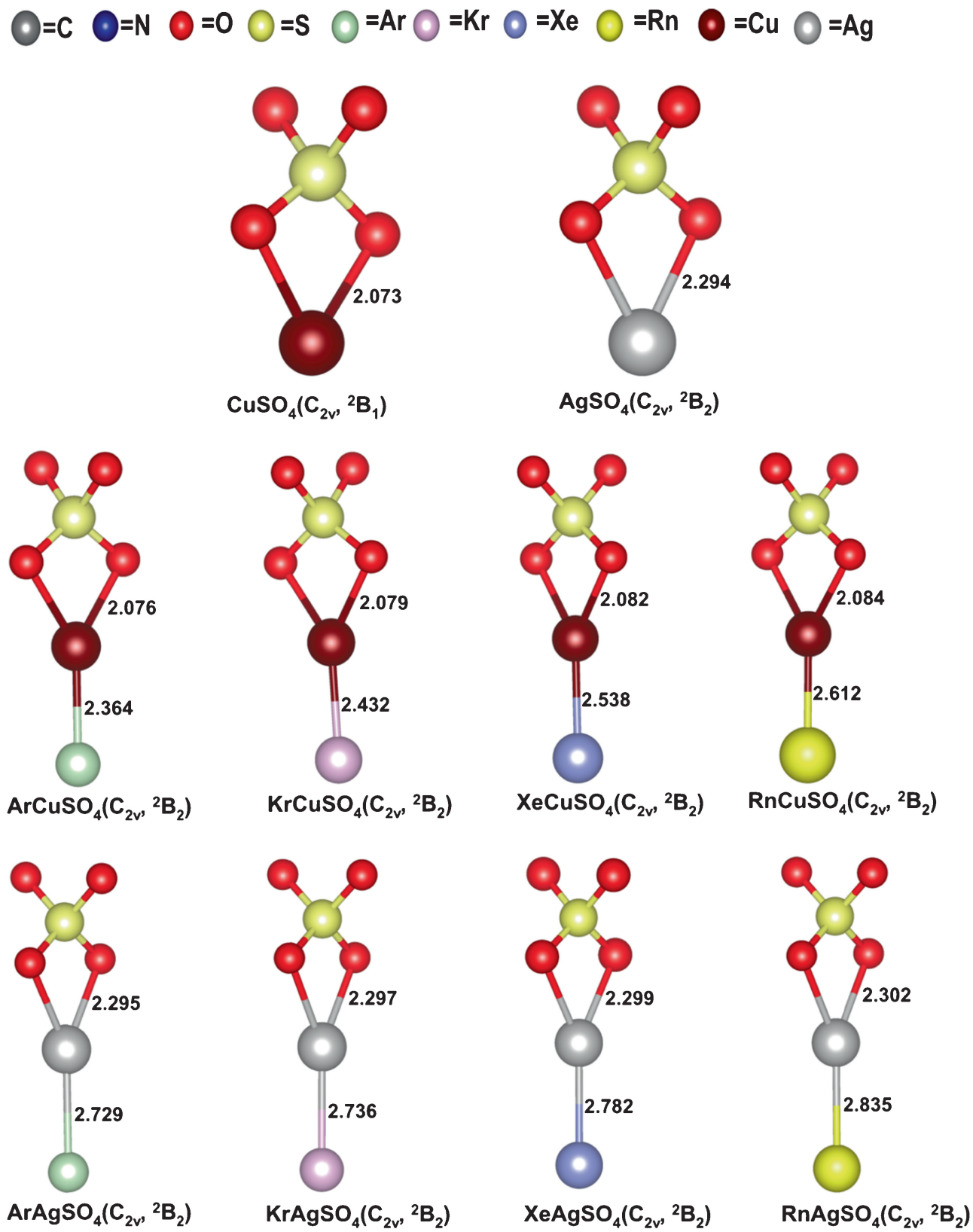

Figure 2. The optimized geometries of $\mathrm{MSO}_{4}$ and their $\mathrm{Ng}$ bound analogues $(\mathrm{M}=\mathrm{Cu}, \mathrm{Ag}$; $\mathrm{Ng}=\mathrm{Ar}-\mathrm{Rn}$ ) at the MPW1B95/def2-TZVP level. Point groups along with their electronic states are given in the parentheses. The $\mathrm{Ng}-\mathrm{M}$ and $\mathrm{M}-\mathrm{O}$ bond distances are given in $\AA$ unit. 
tionary points reported in this study. NPA and $\mathrm{WBIs}^{27}$ are evaluated to get the charge (q) at each atomic center and the Ng-M bond order, respectively. All these calculations are carried out with the Gaussian 09 program package. ${ }^{28}$ The electron density analysis ${ }^{24}$ is performed using Multiwfn software ${ }^{29}$ at the MPW1B95/ def2-TZVP level in order to understand the nature of interaction.

\section{Results and Discussion}

The minimum energy structures of $\mathrm{NgCuNO}_{3}$ correspond to the $C_{2 \mathrm{v}}$ point group symmetry with ${ }^{1} \mathrm{~A}_{1}$ electronic state, whereas in cases of $\mathrm{NgAgNO}_{3}, \mathrm{Ng}$ atoms bind with $\mathrm{Ag}$ in slightly tilted fashion making two $\angle \mathrm{Ng}-\mathrm{Ag}-\mathrm{O}$ bond angles unequal. $\mathrm{NgAgNO}_{3}$ compounds correspond to $C_{\mathrm{s}}$ point group symmetry

Color Code:

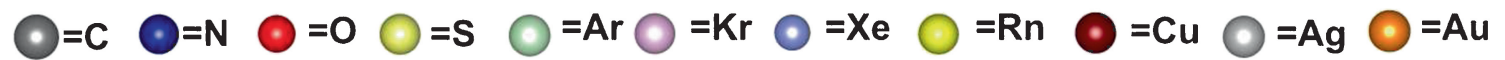
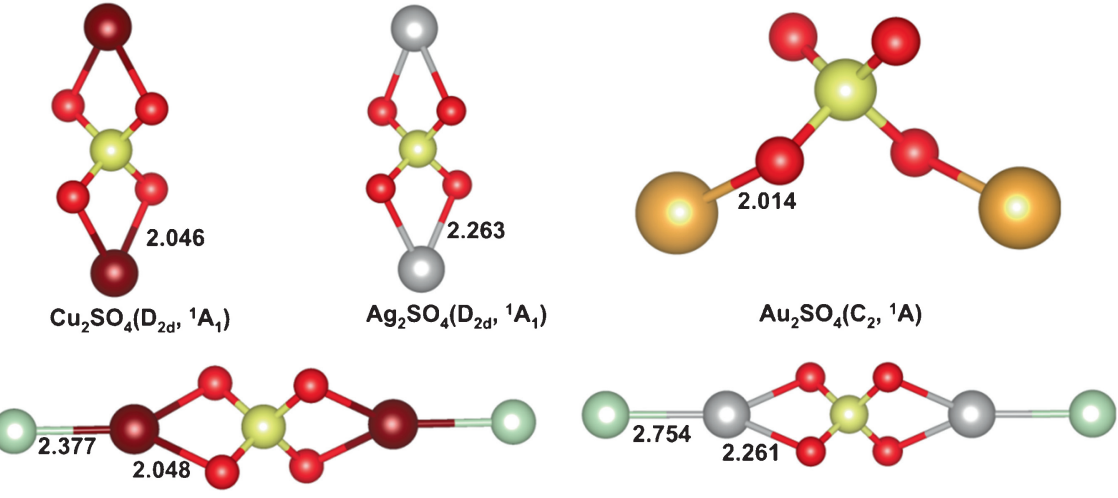

$\mathrm{Ar}_{2} \mathrm{Cu}_{2} \mathrm{SO}_{4}\left(\mathrm{D}_{2 \mathrm{~d}},{ }^{1} \mathrm{~A}_{1}\right)$

$\mathrm{Ar}_{2} \mathrm{Ag}_{2} \mathrm{SO}_{4}\left(\mathrm{D}_{2 \mathrm{~d}},{ }^{1} \mathrm{~A}_{1}\right)$
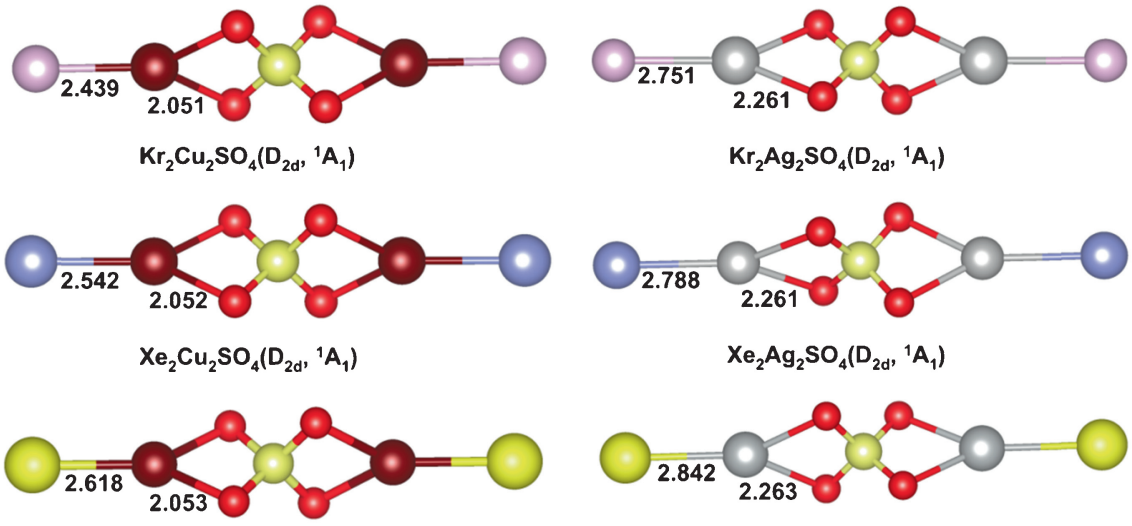

$\mathrm{Rn}_{2} \mathrm{Cu}_{2} \mathrm{SO}_{4}\left(\mathrm{D}_{2 \mathrm{~d}},{ }^{1} \mathrm{~A}_{1}\right)$

$\mathrm{Rn}_{2} \mathrm{Ag}_{2} \mathrm{SO}_{4}\left(\mathrm{D}_{2 \mathrm{~d}},{ }^{1} \mathrm{~A}_{1}\right)$
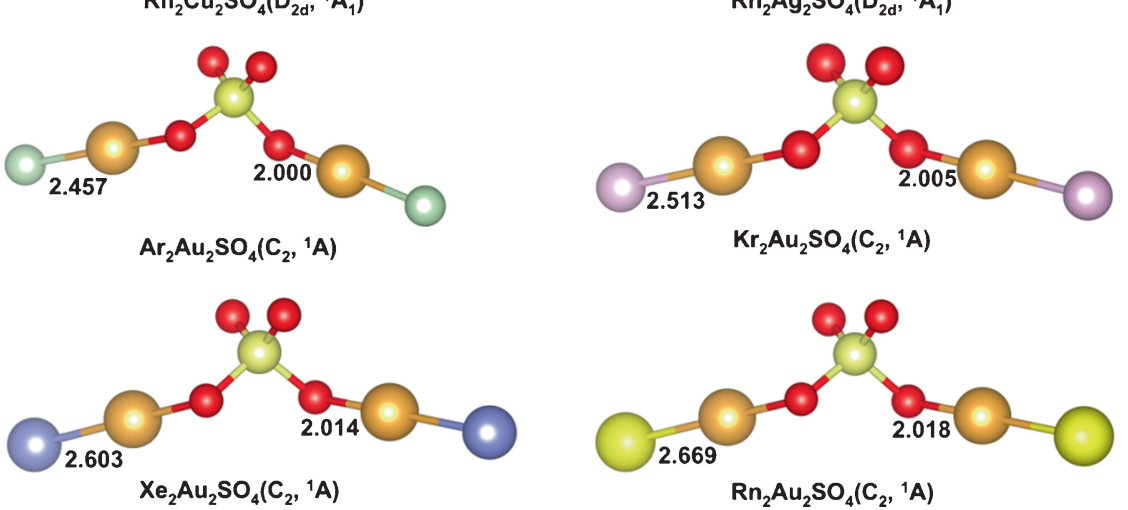

Figure 3. The optimized geometries of $\mathrm{M}_{2} \mathrm{SO}_{4}$ and their $\mathrm{Ng}$ bound analogues $(\mathrm{M}=\mathrm{Cu}, \mathrm{Ag}, \mathrm{Au} ; \mathrm{Ng}=$ $\mathrm{Ar}-\mathrm{Rn}$ ) at the MPW1B95/def2-TZVP level. Point groups along with their electronic states are given in the parentheses. The Ng-M and M-O bond distances are given in $\AA$ unit. 
Color Code:

$\odot=\mathbf{C} \odot=\mathrm{n} \odot=\mathrm{O} \odot=\mathrm{s} \odot=\mathrm{Ar} \odot=\mathrm{kr} \odot=\mathrm{Xe} \odot=\mathrm{Rn} \odot=\mathrm{Cu} \odot=\mathrm{Ag} \odot=\mathrm{Au}$
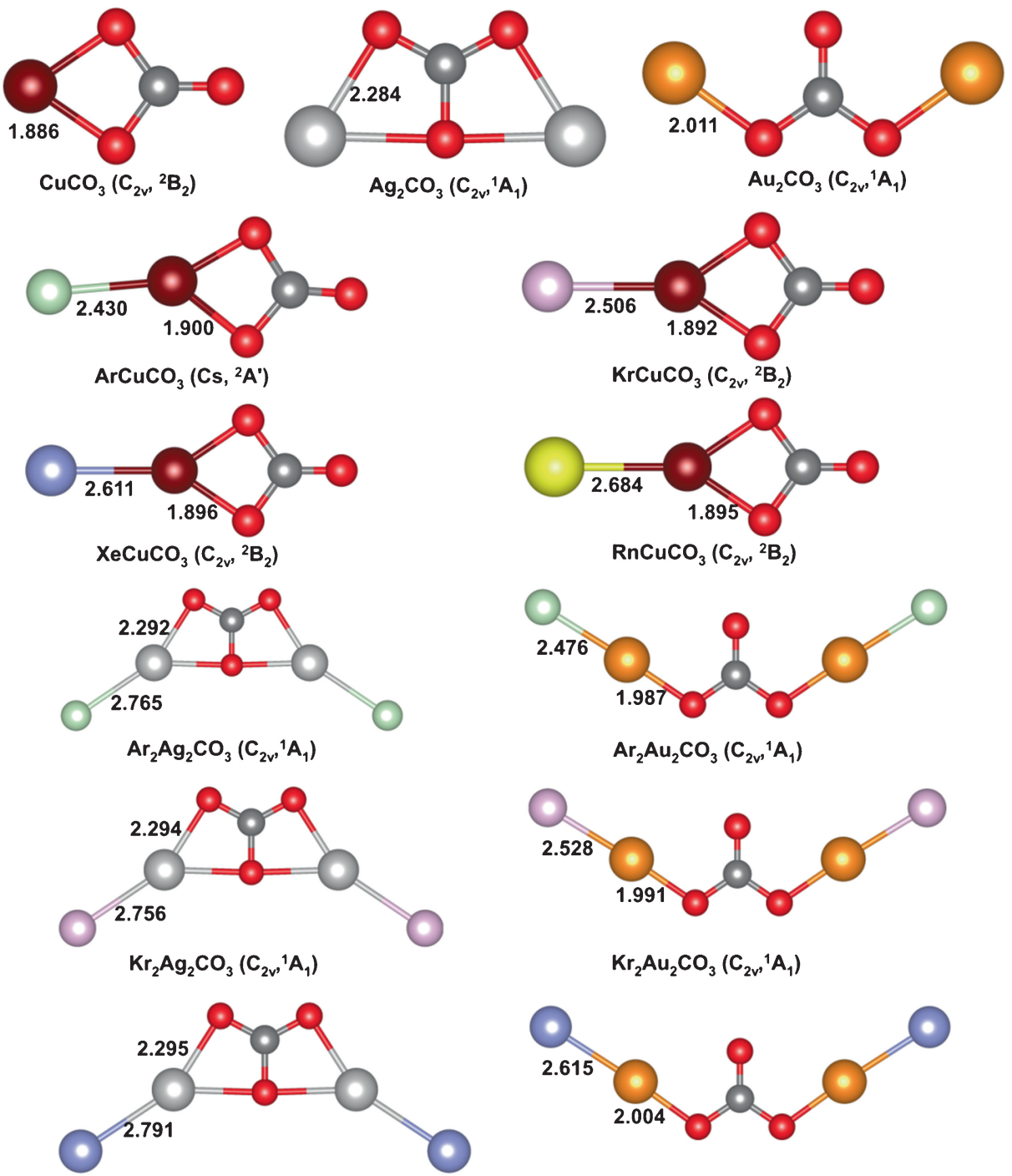

$\mathrm{Kr}_{2} \mathrm{Au}_{2} \mathrm{CO}_{3}\left(\mathrm{C}_{2 v}{ }^{1} \mathrm{~A}_{1}\right)$

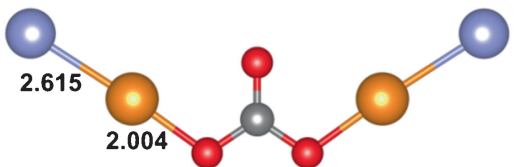

$\mathrm{Xe}_{2} \mathrm{Ag}_{2} \mathrm{CO}_{3}\left(\mathrm{C}_{2 v}{ }^{1} \mathrm{~A}_{1}\right)$

$\mathrm{Xe}_{2} \mathrm{Au}_{2} \mathrm{CO}_{3}\left(\mathrm{C}_{2 v},{ }^{1} \mathrm{~A}_{1}\right)$
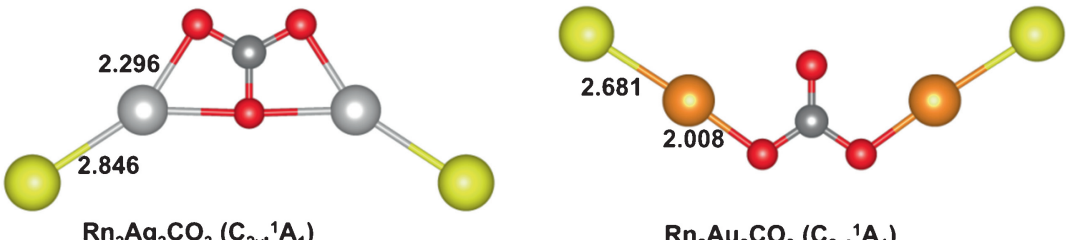

$\operatorname{Rn}_{2} \mathrm{Ag}_{2} \mathrm{CO}_{3}\left(\mathrm{C}_{2 v},{ }^{1} \mathrm{~A}_{1}\right)$

$\mathrm{Rn}_{2} \mathrm{Au}_{2} \mathrm{CO}_{3}\left(\mathrm{C}_{2 v}{ }^{1} \mathrm{~A}_{1}\right)$

Figure 4. The optimized geometries of $\mathrm{CuCO}_{3}, \mathrm{M}_{2} \mathrm{CO}_{3}$ and their $\mathrm{Ng}$ bound analogues $(\mathrm{M}=\mathrm{Ag}, \mathrm{Au} ; \mathrm{Ng}$ $=\mathrm{Ar}-\mathrm{Rn}$ ) at the MPW1B95/def2-TZVP level. Point groups along with their electronic states are given in the parentheses. The Ng-M and M-O bond distances are given in $\AA$ unit.

with ${ }^{1} \mathrm{~A}^{\prime}$ electronic state (Figure 1). The corresponding $C_{2 \mathrm{v}}$ symmetric structures possess a small imaginary frequency and the corresponding mode is connected to the bending of $\mathrm{Ng}-\mathrm{Ag}-\mathrm{O}$ moieties. However, the $C_{\mathrm{s}}$ and $C_{2 \mathrm{v}}$ symmetric structures are almost isoenergetic. We have also tested the $\mathrm{Ng}$ binding ability of $\mathrm{Cu}\left(\mathrm{NO}_{3}\right)_{2}$ but it is found that it binds $\mathrm{Ng}$ atoms very loosely having $\mathrm{Ng}-\mathrm{Cu}$ bond dissociation energies within the range of $0.7-1.5 \mathrm{kcal} / \mathrm{mol}$. Therefore, related results are provided in the supporting information (see Figure S1 
Table 1. ZPE corrected dissociation energy $\left(\mathrm{D}_{0}, \mathrm{kcal} / \mathrm{mol}\right)$ of $\mathrm{Ng}-\mathrm{M}$ bonds, enthalpy change $(\Delta \mathrm{H}, \mathrm{kcal} / \mathrm{mol})$ at $298 \mathrm{~K}$ and free energy change $(\Delta \mathrm{G}, \mathrm{kcal} / \mathrm{mol})$ at $298 \mathrm{~K}$ for the dissociation process: $\mathrm{NgMY} \rightarrow \mathrm{Ng}+\mathrm{MY}\left(\mathrm{M}=\mathrm{Cu}, \mathrm{Ag} ; \mathrm{Y}=\mathrm{NO}_{3}, \mathrm{SO}_{4}\right.$, $\mathrm{CO}_{3}$ ), NPA charge at $\mathrm{M}$ and $\mathrm{Ng}$ centers (q, au), Wiberg bond indices of Ng-M bonds (WBI), HOMO-LUMO energy gaps (Gap, eV), and Ng-M bond distances $\left(\mathrm{r}_{\mathrm{Ng}-\mathrm{M}}, \AA\right)$ at the MPW1B95/def2-TZVP level.

\begin{tabular}{|c|c|c|c|c|c|c|c|c|}
\hline Compounds & $\mathrm{D}_{0}$ & $\Delta \mathrm{H}$ & $\Delta \mathrm{G}$ & $\mathrm{q}(\mathrm{M})$ & $\mathrm{q}(\mathrm{Ng})$ & WBI & Gap & $\mathrm{r}_{\mathrm{Ng}-\mathrm{M}}$ \\
\hline $\mathrm{CuNO}_{3}$ & & & & 0.81 & & & 4.574 & \\
\hline $\mathrm{ArCuNO}_{3}$ & 6.0 & 6.0 & 0.1 & 0.68 & 0.09 & 0.19 & 5.837 & 2.356 \\
\hline $\mathrm{KrCuNO}_{3}$ & 8.7 & 8.6 & 3.2 & 0.64 & 0.12 & 0.25 & 5.974 & 2.427 \\
\hline $\mathrm{XeCuNO}_{3}$ & 12.2 & 12.2 & 6.4 & 0.59 & 0.17 & 0.34 & 6.078 & 2.534 \\
\hline $\mathrm{RnCuNO}_{3}$ & 13.1 & 13.0 & 7.6 & 0.58 & 0.17 & 0.35 & 5.877 & 2.608 \\
\hline $\mathrm{AgNO}_{3}$ & & & & 0.83 & & & 4.198 & \\
\hline $\mathrm{ArAgNO}_{3}$ & 3.1 & 3.0 & -1.8 & 0.75 & 0.05 & 0.10 & 4.911 & 2.725 \\
\hline $\mathrm{KrAgNO}_{3}$ & 5.2 & 5.1 & -0.5 & 0.71 & 0.08 & 0.16 & 5.108 & 2.734 \\
\hline $\mathrm{XeAgNO}$ & 8.5 & 8.4 & 3.1 & 0.65 & 0.13 & 0.26 & 5.312 & 2.776 \\
\hline $\mathrm{RnAgNO}_{3}$ & 9.8 & 9.7 & 4.4 & 0.64 & 0.14 & 0.28 & 5.201 & 2.830 \\
\hline $\mathrm{CuSO}_{4}$ & & & & 0.86 & & & 4.100 & \\
\hline $\mathrm{ArCuSO}_{4}$ & 6.0 & 6.0 & -0.3 & 0.73 & 0.09 & 0.19 & 4.717 & 2.364 \\
\hline $\mathrm{KrCuSO}_{4}$ & 8.7 & 8.7 & 2.3 & 0.69 & 0.12 & 0.25 & 4.796 & 2.432 \\
\hline $\mathrm{XeCuSO}_{4}$ & 12.3 & 12.3 & 5.8 & 0.63 & 0.17 & 0.34 & 4.856 & 2.538 \\
\hline $\mathrm{RnCuSO}_{4}$ & 13.2 & 13.2 & 6.7 & 0.63 & 0.18 & 0.35 & 4.747 & 2.612 \\
\hline $\mathrm{AgSO}_{4}$ & & & & 0.88 & & & 4.327 & \\
\hline $\mathrm{ArAgSO}_{4}$ & 3.2 & 3.1 & -2.6 & 0.81 & 0.05 & 0.11 & 4.656 & 2.729 \\
\hline $\mathrm{KrAgSO}_{4}$ & 5.3 & 5.2 & -0.3 & 0.77 & 0.08 & 0.17 & 4.757 & 2.736 \\
\hline $\mathrm{XeAgSO}_{4}$ & 8.7 & 8.6 & 3.0 & 0.71 & 0.13 & 0.26 & 4.853 & 2.782 \\
\hline $\mathrm{RnAgSO}_{4}$ & 10.1 & 9.9 & 4.9 & 0.69 & 0.15 & 0.29 & 4.801 & 2.835 \\
\hline $\mathrm{CuCO}_{3}$ & & & & 1.02 & & & 4.296 & \\
\hline $\mathrm{ArCuCO}_{3}$ & 5.7 & 5.7 & 0.2 & 0.89 & 0.09 & 0.18 & 4.898 & 2.430 \\
\hline $\mathrm{KrCuCO}_{3}$ & 8.1 & 8.0 & 2.3 & 0.84 & 0.13 & 0.24 & 4.970 & 2.506 \\
\hline $\mathrm{XeCuCO}_{3}$ & 11.3 & 11.2 & 5.3 & 0.79 & 0.17 & 0.33 & 5.010 & 2.611 \\
\hline $\mathrm{RnCuCO}_{3}$ & 12.4 & 12.3 & 6.4 & 0.78 & 0.19 & 0.34 & 4.921 & 2.684 \\
\hline
\end{tabular}

Table 2. ZPE corrected dissociation energy $\left(\mathrm{D}_{0}, \mathrm{kcal} / \mathrm{mol}\right)$ of per $\mathrm{Ng}-\mathrm{M}$ bonds, enthalpy change $(\Delta \mathrm{H}, \mathrm{kcal} / \mathrm{mol})$ at $298 \mathrm{~K}$ and free energy change $(\Delta \mathrm{G}, \mathrm{kcal} / \mathrm{mol})$ at $298 \mathrm{~K}$ for the dissociation process: $\mathrm{Ng}_{2} \mathrm{M}_{2} \mathrm{Y} \rightarrow 2 \mathrm{Ng}+\mathrm{M}_{2} \mathrm{Y}(\mathrm{M}=\mathrm{Cu}, \mathrm{Ag}, \mathrm{Au}$; $\mathrm{Y}=\mathrm{SO}_{4}, \mathrm{CO}_{3}$ ), NPA charge at $\mathrm{M}$ and $\mathrm{Ng}$ centers (q, au), Wiberg bond indices of Ng-M bonds (WBI), HOMO-LUMO energy gaps (Gap, eV), and Ng-M bond distances ( $\left.\mathrm{r}_{\mathrm{Ng}-\mathrm{M}}, \AA\right)$ at the MPW1B95/def2-TZVP level.

\begin{tabular}{lcccccccr}
\hline Compounds & $\mathrm{D}_{0}$ & $\Delta \mathrm{H}$ & $\Delta \mathrm{G}$ & $\mathrm{q}(\mathrm{M})$ & $\mathrm{q}(\mathrm{Ng})$ & WBI & Gap & $\mathrm{r}_{\mathrm{Ng}-\mathrm{M}}$ \\
\hline $\mathrm{Cu}_{2} \mathrm{SO}_{4}$ & & & & 0.84 & & & 4.574 & \\
$\mathrm{Ar}_{2} \mathrm{Cu}_{2} \mathrm{SO}_{4}$ & 5.1 & 10.2 & -2.0 & 0.71 & 0.09 & 0.18 & 5.714 & 2.377 \\
$\mathrm{Kr}_{2} \mathrm{Cu}_{2} \mathrm{SO}_{4}$ & 7.6 & 15.1 & 2.7 & 0.67 & 0.12 & 0.25 & 5.862 & 2.439 \\
$\mathrm{Xe}_{2} \mathrm{Cu}_{2} \mathrm{SO}_{4}$ & 11.0 & 21.9 & 8.9 & 0.61 & 0.16 & 0.33 & 5.975 & 2.542 \\
$\mathrm{Rn}_{2} \mathrm{Cu}_{2} \mathrm{SO}_{4}$ & 11.7 & 23.2 & 10.2 & 0.61 & 0.17 & 0.33 & 5.778 & 2.618 \\
$\mathrm{Ag}_{2} \mathrm{SO}_{4}$ & & & & 0.85 & & & & \\
$\mathrm{Ar}_{2} \mathrm{Ag}_{2} \mathrm{SO}_{4}$ & 2.5 & 4.9 & -6.8 & 0.78 & 0.05 & 0.10 & 4.866 & 2.754 \\
$\mathrm{Kr}_{2} \mathrm{Ag}_{2} \mathrm{SO}_{4}$ & 4.4 & 8.6 & -3.2 & 0.74 & 0.08 & 0.16 & 5.058 & 2.751 \\
$\mathrm{Xe}_{2} \mathrm{Ag}_{2} \mathrm{SO}_{4}$ & 7.5 & 14.7 & 2.6 & 0.68 & 0.12 & 0.25 & 5.245 & 2.788 \\
$\mathrm{Rn}_{2} \mathrm{Ag}_{2} \mathrm{SO}_{4}$ & 8.6 & 16.9 & 4.8 & 0.67 & 0.14 & 0.27 & 5.118 & 2.842 \\
$\mathrm{Au}_{2} \mathrm{SO}_{4}$ & & & & 0.66 & & & 3.884 & \\
$\mathrm{Ar}_{2} \mathrm{Au}_{2} \mathrm{SO}_{4}$ & 8.1 & 16.5 & 1.8 & 0.57 & 0.11 & 0.21 & 5.294 & 2.457 \\
$\mathrm{Kr}_{2} \mathrm{Au}_{2} \mathrm{SO}_{4}$ & 12.5 & 25.2 & 10.0 & 0.53 & 0.16 & 0.30 & 5.439 & 2.513 \\
$\mathrm{Xe}_{2} \mathrm{Au}_{2} \mathrm{SO}_{4}$ & 18.3 & 36.7 & 21.4 & 0.48 & 0.22 & 0.41 & 5.477 & 2.603 \\
$\mathrm{Rn}_{2} \mathrm{Au}_{2} \mathrm{SO}_{4}$ & 19.9 & 39.9 & 24.7 & 0.46 & 0.24 & 0.43 & 5.302 & 2.669 \\
$\mathrm{Ag}_{2} \mathrm{CO}_{3}$ & & & & 0.81 & & & 3.355 & \\
$\mathrm{Ar}_{2} \mathrm{Ag}_{2} \mathrm{CO}_{3}$ & 2.3 & 4.4 & -7.1 & 0.74 & 0.05 & 0.09 & 3.941 & 2.765 \\
$\mathrm{Kr}_{2} \mathrm{Ag}_{2} \mathrm{CO}_{3}$ & 4.1 & 8.0 & -4.1 & 0.70 & 0.07 & 0.15 & 4.127 & 2.756 \\
$\mathrm{Xe}_{2} \mathrm{Ag}_{2} \mathrm{CO}_{3}$ & 7.0 & 13.8 & 1.4 & 0.65 & 0.12 & 0.25 & 4.307 & 2.791 \\
$\mathrm{Rn}_{2} \mathrm{Ag}_{2} \mathrm{CO}_{3}$ & 8.0 & 15.9 & 3.4 & 0.63 & 0.13 & 0.26 & 4.177 & 2.846
\end{tabular}


Table 2. Continued.

\begin{tabular}{lcccccccr}
\hline Compounds & $\mathrm{D}_{0}$ & $\Delta \mathrm{H}$ & $\Delta \mathrm{G}$ & $\mathrm{q}(\mathrm{M})$ & $\mathrm{q}(\mathrm{Ng})$ & WBI & Gap & $\mathrm{r}_{\mathrm{Ng}-\mathrm{M}}$ \\
\hline $\mathrm{Au}_{2} \mathrm{CO}_{3}$ & & & & 0.62 & & & 3.185 & \\
$\mathrm{Ar}_{2} \mathrm{Au}_{2} \mathrm{CO}_{3}$ & 7.3 & 15.0 & 0.1 & 0.52 & 0.11 & 0.20 & 4.595 & 2.476 \\
$\mathrm{Kr}_{2} \mathrm{Au}_{2} \mathrm{CO}_{3}$ & 11.4 & 22.9 & 7.9 & 0.49 & 0.15 & 0.27 & 4.760 & 2.528 \\
$\mathrm{Xe}_{2} \mathrm{Au}_{2} \mathrm{CO}_{3}$ & 16.7 & 33.5 & 18.6 & 0.44 & 0.21 & 0.38 & 4.786 & 2.615 \\
$\mathrm{Rn}_{2} \mathrm{Au}_{2} \mathrm{CO}_{3}$ & 18.2 & 36.5 & 21.6 & 0.43 & 0.22 & 0.40 & 4.604 & 2.681 \\
\hline
\end{tabular}

Table 3. Different topological descriptors (au) at the bond critical points (BCP) in between $\mathrm{Ng}$ and $\mathrm{M}$ atoms in $\mathrm{NgMY}$ (M $=\mathrm{Cu}, \mathrm{Ag} ; \mathrm{Y}=\mathrm{NO}_{3}, \mathrm{SO}_{4}, \mathrm{CO}_{3}$ ) at the MPW1B95/def2-TZVP level.

\begin{tabular}{|c|c|c|c|c|c|c|}
\hline Compounds & $\mathrm{BCP}$ & $\rho\left(\mathbf{r}_{\mathbf{c}}\right)$ & $\nabla^{2} \rho\left(\mathbf{r}_{\mathbf{c}}\right)$ & $\mathrm{G}\left(\mathbf{r}_{\mathbf{c}}\right)$ & $\mathrm{V}\left(\mathbf{r}_{\mathbf{c}}\right)$ & $\mathrm{H}\left(\mathbf{r}_{\mathbf{c}}\right)$ \\
\hline $\mathrm{ArCuNO}_{3}$ & $\mathrm{Ar}-\mathrm{Cu}$ & 0.046 & 0.231 & 0.062 & -0.067 & -0.004 \\
\hline $\mathrm{KrCuNO}_{3}$ & $\mathrm{Kr}-\mathrm{Cu}$ & 0.050 & 0.209 & 0.059 & -0.066 & -0.007 \\
\hline $\mathrm{XeCuNO}_{3}$ & $\mathrm{Xe}-\mathrm{Cu}$ & 0.052 & 0.173 & 0.053 & -0.062 & -0.009 \\
\hline $\mathrm{RnCuNO}_{3}$ & $\mathrm{Rn}-\mathrm{Cu}$ & 0.050 & 0.150 & 0.046 & -0.055 & -0.009 \\
\hline $\mathrm{ArAgNO}_{3}$ & $\mathrm{Ar}-\mathrm{Ag}$ & 0.028 & 0.122 & 0.031 & -0.031 & 0.000 \\
\hline $\mathrm{KrAgNO}_{3}$ & $\mathrm{Kr}-\mathrm{Ag}$ & 0.036 & 0.129 & 0.035 & -0.038 & -0.003 \\
\hline $\mathrm{XeAgNO}_{3}$ & $\mathrm{Xe}-\mathrm{Ag}$ & 0.043 & 0.128 & 0.038 & -0.043 & -0.006 \\
\hline $\mathrm{RnAgNO}_{3}$ & $\mathrm{Rn}-\mathrm{Ag}$ & 0.043 & 0.115 & 0.035 & -0.042 & -0.006 \\
\hline $\mathrm{ArCuSO}_{4}$ & $\mathrm{Ar}-\mathrm{Cu}$ & 0.045 & 0.227 & 0.061 & -0.065 & -0.004 \\
\hline $\mathrm{KrCuSO}_{4}$ & $\mathrm{Kr}-\mathrm{Cu}$ & 0.050 & 0.206 & 0.059 & -0.066 & -0.007 \\
\hline $\mathrm{XeCuSO}_{4}$ & $\mathrm{Xe}-\mathrm{Cu}$ & 0.053 & 0.171 & 0.052 & -0.062 & -0.009 \\
\hline $\mathrm{RnCuSO}_{4}$ & $\mathrm{Rn}-\mathrm{Cu}$ & 0.050 & 0.149 & 0.046 & -0.055 & -0.009 \\
\hline $\mathrm{ArAgSO}_{4}$ & $\mathrm{Ar}-\mathrm{Ag}$ & 0.028 & 0.121 & 0.030 & -0.031 & 0.000 \\
\hline $\mathrm{KrAgSO}_{4}$ & $\mathrm{Kr}-\mathrm{Ag}$ & 0.036 & 0.129 & 0.035 & -0.038 & -0.003 \\
\hline $\mathrm{XeAgSO}_{4}$ & $\mathrm{Xe}-\mathrm{Ag}$ & 0.043 & 0.126 & 0.037 & -0.043 & -0.006 \\
\hline $\mathrm{RnAgSO}_{4}$ & $\mathrm{Rn}-\mathrm{Ag}$ & 0.043 & 0.113 & 0.035 & -0.041 & -0.007 \\
\hline $\mathrm{ArCuCO}_{3}$ & $\mathrm{Ar}-\mathrm{Cu}$ & 0.039 & 0.188 & 0.049 & -0.051 & -0.002 \\
\hline $\mathrm{KrCuCO}_{3}$ & $\mathrm{Kr}-\mathrm{Cu}$ & 0.043 & 0.170 & 0.047 & -0.051 & -0.005 \\
\hline $\mathrm{XeCuCO}_{3}$ & $\mathrm{Xe}-\mathrm{Cu}$ & 0.046 & 0.144 & 0.043 & -0.049 & -0.007 \\
\hline $\mathrm{RnCuCO}_{3}$ & $\mathrm{Rn}-\mathrm{Cu}$ & 0.044 & 0.125 & 0.038 & -0.044 & -0.007 \\
\hline
\end{tabular}

and Table S1 in Supplementary Information). In cases of sulphates, while all the $\mathrm{Ng}$ bound $\mathrm{CuSO}_{4}$ and $\mathrm{AgSO}_{4}$ geometries correspond to a $C_{2 v}$ point group and ${ }^{2} \mathrm{~B}_{2}$ electronic state, the structures of $\mathrm{Ng}_{2} \mathrm{Cu}_{2} \mathrm{SO}_{4}$ and $\mathrm{Ng}_{2} \mathrm{Ag}_{2} \mathrm{SO}_{4}$ belong to a $D_{2 d}$ symmetry with ${ }^{1} \mathrm{~A}_{1}$ electronic state (see Figures 2 and 3). In contrast, the optimized structures of $\mathrm{Ng}_{2} \mathrm{Au}_{2} \mathrm{SO}_{4}$ correspond to $C_{2}$ symmetry and ${ }^{1} \mathrm{~A}$ electronic state. It may be noted that the geometry of $\mathrm{Au}_{2} \mathrm{SO}_{4}$ is slightly different from those of its lighter homologues. While in $\mathrm{Cu}_{2} \mathrm{SO}_{4}$ and $\mathrm{Ag}_{2} \mathrm{SO}_{4}, \mathrm{M}$ centers occupy a position in between two $\mathrm{O}$ centers of $\mathrm{SO}_{4}$ unit providing the structure a $D_{2 d}$ symmetry, in $\mathrm{Au}_{2} \mathrm{SO}_{4}$ the $\mathrm{Au}$ atoms bind with only one $\mathrm{O}$ center of $\mathrm{SO}_{4}$ yielding a $C_{2}$ symmetric structure. Again the geometry of $\mathrm{NgCuCO}_{3}$ has a $C_{2 v}$ symmetry with ${ }^{2} \mathrm{~B}_{2}$ electronic state except for $\mathrm{ArCuCO}_{3}$ which belongs to a $C_{s}$ symmetry and ${ }^{2} \mathrm{~A}^{\prime}$ electronic state. The $C_{2 v}$ symmetric $\mathrm{ArCuCO}_{3}$ contains an imaginary frequency which leads to the two unequal $\angle \mathrm{Ar}-\mathrm{Cu}-\mathrm{O}$ bond angles (Figure 4). Moreover, the minimum energy structures of all $\mathrm{Ng}_{2} \mathrm{Ag}_{2} \mathrm{CO}_{3}$ and $\mathrm{Ng}_{2} \mathrm{Au}_{2} \mathrm{CO}_{3}$ correspond to a $C_{2 v}$ symmetry and ${ }^{1} \mathrm{~A}_{1}$ electronic states (Figure 4).

The results of ZPE corrected bond dissociation energy $\left(D_{0}\right)$, thermochemical parameters like $\Delta H$ and $\Delta \mathrm{G}$ as well as the NPA charge on metal (M) and $\mathrm{Ng}$ centers and WBI values for $\mathrm{Ng}-\mathrm{M}$ bonds in $\mathrm{NgMY}$ $\left(\mathrm{Y}=\mathrm{NO}_{3}, \mathrm{SO}_{4}\right)$ and $\mathrm{NgCuCO}_{3}$ are provided in Table 1. All the thermochemical parameters are computed at one atmospheric pressure and $298 \mathrm{~K}$. Since $\mathrm{He}$ and $\mathrm{Ne}$ bind with $\mathrm{M}$ center very loosely, those results are not included here. The $\mathrm{D}_{0}$ values for the $\mathrm{Ng}-\mathrm{M}$ bonds range within $6.0-13.1 \mathrm{kcal} / \mathrm{mol}$ in $\mathrm{NgCuNO}_{3}, 3.1-9.8 \mathrm{kcal} / \mathrm{mol}$ in $\mathrm{NgAgNO}_{3}, 6.0-13.2 \mathrm{kcal} / \mathrm{mol}$ in $\mathrm{NgCuSO}_{4}, 3.2-10.1$ $\mathrm{kcal} / \mathrm{mol}$ in $\mathrm{NgAgSO}_{4}$ and $5.7-12.4 \mathrm{kcal} / \mathrm{mol}$ in $\mathrm{NgCuCO}_{3}$ with gradual increase along $\mathrm{Ar}$ to $\mathrm{Rn}$. This 
Table 4. Different topological descriptors (au) at the bond critical points (BCP) in between $\mathrm{Ng}$ and $\mathrm{M}$ atoms in $\mathrm{Ng}_{2} \mathrm{M}_{2} \mathrm{Y}$ $\left(\mathrm{M}=\mathrm{Cu}, \mathrm{Ag}, \mathrm{Au} ; \mathrm{Y}=\mathrm{SO}_{4}, \mathrm{CO}_{3}\right)$ at the MPW1B95/def2-TZVP level.

\begin{tabular}{|c|c|c|c|c|c|c|}
\hline Compounds & $\mathrm{BCP}$ & $\rho\left(\mathbf{r}_{\mathbf{c}}\right)$ & $\nabla^{2} \rho\left(\mathbf{r}_{\mathbf{c}}\right)$ & $\mathrm{G}\left(\mathbf{r}_{\mathbf{c}}\right)$ & $\mathrm{V}\left(\mathbf{r}_{\mathbf{c}}\right)$ & $\mathrm{H}\left(\mathbf{r}_{\mathbf{c}}\right)$ \\
\hline $\mathrm{Ar}_{2} \mathrm{Cu}_{2} \mathrm{SO}_{4}$ & $\mathrm{Ar}-\mathrm{Cu}$ & 0.044 & 0.221 & 0.059 & -0.062 & -0.004 \\
\hline $\mathrm{Kr}_{2} \mathrm{Cu}_{2} \mathrm{SO}_{4}$ & $\mathrm{Kr}-\mathrm{Cu}$ & 0.049 & 0.205 & 0.058 & -0.064 & -0.007 \\
\hline $\mathrm{Xe}_{2} \mathrm{Cu}_{2} \mathrm{SO}_{4}$ & $\mathrm{Xe}-\mathrm{Cu}$ & 0.052 & 0.172 & 0.052 & -0.061 & -0.009 \\
\hline $\mathrm{Rn}_{2} \mathrm{Cu}_{2} \mathrm{SO}_{4}$ & $\mathrm{Rn}-\mathrm{Cu}$ & 0.049 & 0.149 & 0.046 & -0.054 & -0.008 \\
\hline $\mathrm{Ar}_{2} \mathrm{Ag}_{2} \mathrm{SO}_{4}$ & Ar-Ag & 0.026 & 0.114 & 0.028 & -0.028 & 0.000 \\
\hline $\mathrm{Kr}_{2} \mathrm{Ag}_{2} \mathrm{SO}_{4}$ & $\mathrm{Kr}-\mathrm{Ag}$ & 0.034 & 0.125 & 0.034 & -0.037 & -0.003 \\
\hline $\mathrm{Xe}_{2} \mathrm{Ag}_{2} \mathrm{SO}_{4}$ & $\mathrm{Xe}-\mathrm{Ag}$ & 0.042 & 0.126 & 0.037 & -0.042 & -0.005 \\
\hline $\mathrm{Rn}_{2} \mathrm{Ag}_{2} \mathrm{SO}_{4}$ & $\mathrm{Rn}-\mathrm{Ag}$ & 0.042 & 0.113 & 0.034 & -0.041 & -0.006 \\
\hline $\mathrm{Ar}_{2} \mathrm{Au}_{2} \mathrm{SO}_{4}$ & $\mathrm{Ar}-\mathrm{Au}$ & 0.058 & 0.242 & 0.069 & -0.078 & -0.009 \\
\hline $\mathrm{Kr}_{2} \mathrm{Au}_{2} \mathrm{SO}_{4}$ & $\mathrm{Kr}-\mathrm{Au}$ & 0.066 & 0.216 & 0.067 & -0.080 & -0.013 \\
\hline $\mathrm{Xe}_{2} \mathrm{Au}_{2} \mathrm{SO}_{4}$ & $\mathrm{Xe}-\mathrm{Au}$ & 0.071 & 0.166 & 0.060 & -0.078 & -0.018 \\
\hline $\mathrm{Rn}_{2} \mathrm{Au}_{2} \mathrm{SO}_{4}$ & $\mathrm{Rn}-\mathrm{Au}$ & 0.068 & 0.143 & 0.054 & -0.072 & -0.018 \\
\hline $\mathrm{Ar}_{2} \mathrm{Ag}_{2} \mathrm{CO}_{3}$ & $\mathrm{Ar}-\mathrm{Ag}$ & 0.026 & 0.111 & 0.028 & -0.027 & 0.000 \\
\hline $\mathrm{Kr}_{2} \mathrm{Ag}_{2} \mathrm{CO}_{3}$ & $\mathrm{Kr}-\mathrm{Ag}$ & 0.034 & 0.124 & 0.033 & -0.036 & -0.003 \\
\hline $\mathrm{Xe}_{2} \mathrm{Ag}_{2} \mathrm{CO}_{3}$ & $\mathrm{Xe}-\mathrm{Ag}$ & 0.042 & 0.125 & 0.037 & -0.042 & -0.005 \\
\hline $\mathrm{Rn}_{2} \mathrm{Ag}_{2} \mathrm{CO}_{3}$ & $\mathrm{Rn}-\mathrm{Ag}$ & 0.041 & 0.112 & 0.034 & -0.040 & -0.006 \\
\hline $\mathrm{Ar}_{2} \mathrm{Au}_{2} \mathrm{CO}_{3}$ & $\mathrm{Ar}-\mathrm{Au}$ & 0.055 & 0.234 & 0.066 & -0.073 & -0.007 \\
\hline $\mathrm{Kr}_{2} \mathrm{Au}_{2} \mathrm{CO}_{3}$ & $\mathrm{Kr}-\mathrm{Au}$ & 0.063 & 0.212 & 0.065 & -0.077 & -0.012 \\
\hline $\mathrm{Xe}_{2} \mathrm{Au}_{2} \mathrm{CO}_{3}$ & $\mathrm{Xe}-\mathrm{Au}$ & 0.069 & 0.166 & 0.058 & -0.075 & -0.017 \\
\hline $\mathrm{Rn}_{2} \mathrm{Au}_{2} \mathrm{CO}_{3}$ & $\mathrm{Rn}-\mathrm{Au}$ & 0.066 & 0.143 & 0.053 & -0.070 & -0.017 \\
\hline
\end{tabular}

implies that the $\mathrm{Ng}$ atoms with larger atomic masses are bound more strongly with a given system. This is due to the increase in polarizability along the group. It is also found that the $\mathrm{Ng}$ binding ability of $\mathrm{CuY}$ is higher than that of AgY. This is due to the larger polarizing power of $\mathrm{Cu}$ than $\mathrm{Ag}$ as $\mathrm{Cu}$ has smaller size than $\mathrm{Ag}$. The $\mathrm{D}_{0}$ values also reveal that these $\mathrm{MY}$ and $\mathrm{CuCO}_{3}$ have very much comparable $\mathrm{Ng}$ binding ability. All the dissociation processes are endothermic in nature. However, except for $\mathrm{ArCuSO}_{4}, \mathrm{ArAgY}$ and $\mathrm{KrAgY}$ (Y = $\mathrm{SO}_{4}, \mathrm{NO}_{3}$ ) the dissociations of all other $\mathrm{NgMY}(\mathrm{Y}=$ $\mathrm{SO}_{4}, \mathrm{NO}_{3}, \mathrm{CO}_{3}$ ) compounds under consideration are endergonic in nature at room temperature. The $\Delta \mathrm{G}$ values for the dissociations of $\mathrm{KrAgNO}_{3}$ and $\mathrm{KrAgSO}_{4}$ are only slightly negative $(-0.5$ and $-0.3 \mathrm{kcal} / \mathrm{mol}$, respectively) and therefore, they can be made viable at slightly lower temperature.

Now, we have checked the stability of $\mathrm{Ng}_{2} \mathrm{M}_{2} \mathrm{SO}_{4}$ $(\mathrm{M}=\mathrm{Cu}, \mathrm{Ag}, \mathrm{Au})$ and $\mathrm{Ng}_{2} \mathrm{M}_{2} \mathrm{CO}_{3}(\mathrm{M}=\mathrm{Ag}, \mathrm{Au})$ and the related results are provided in Table 2 . The $\mathrm{D}_{0}$ values per $\mathrm{Ng}-\mathrm{M}$ bond lie within the range of $5.1-11.7 \mathrm{kcal} / \mathrm{mol}$ in $\mathrm{Ng}_{2} \mathrm{Cu}_{2} \mathrm{SO}_{4}, 2.5-8.6 \mathrm{kcal} / \mathrm{mol}$ in $\mathrm{Ng}_{2} \mathrm{Ag}_{2} \mathrm{SO}_{4}, 8.1-$ $19.9 \mathrm{kcal} / \mathrm{mol}$ in $\mathrm{Ng}_{2} \mathrm{Au}_{2} \mathrm{SO}_{4}, \quad 2.3-8.0 \mathrm{kcal} / \mathrm{mol}$ in $\mathrm{Ng}_{2} \mathrm{Ag}_{2} \mathrm{CO}_{3}$ and 7.3-18.2 in $\mathrm{Ng}_{2} \mathrm{Au}_{2} \mathrm{CO}_{3}$ with a gradual increase in moving from $\mathrm{Ar}$ to $\mathrm{Rn}$. The corresponding dissociation processes producing $\mathrm{Ngs}$ and $\mathrm{M}_{2} \mathrm{Y}$ are found to be spontaneous only in cases of $\mathrm{Ar}_{2} \mathrm{Cu}_{2} \mathrm{SO}_{4}$,
$\mathrm{Ar}_{2} \mathrm{Ag}_{2} \mathrm{Y}$ and $\mathrm{Kr}_{2} \mathrm{Ag}_{2} \mathrm{Y}\left(\mathrm{Y}=\mathrm{SO}_{4}\right.$ and $\left.\mathrm{CO}_{3}\right)$. The rest of the systems are viable even at room temperature.

According to maximum hardness principle, ${ }^{30}$ a molecule with higher HOMO-LUMO energy gap is associated with greater stability and hence less reactivity. The related energy gap is found to increase considerably in all $\mathrm{Ng}$ bound cases, implying additional stability in $\mathrm{Ng}$ bound $\mathrm{MY}$ and $\mathrm{M}_{2} \mathrm{Y}$ systems compared to the corresponding bare structures (Tables 1 and 2).

NPA reveals that transfer of electronic charge takes place from $\mathrm{Ng}$ to the $\mathrm{M}$ center in $\mathrm{NgMY}$ and $\mathrm{Ng}_{2} \mathrm{M}_{2} \mathrm{Y}$. The degree of electron transfer is low for lighter $\mathrm{Ng}$ cases in comparison to heavier $\mathrm{Ng}$ atoms. Also, the trend in electron transfer from $\mathrm{Ng}$ centers follows the order of $\mathrm{Rn}>\mathrm{Xe}>\mathrm{Kr}>\mathrm{Ar}$ as the ionization energy increases in reverse order. Furthermore, the extent of electron transfer from a given $\mathrm{Ng}$ atom is the largest when $\mathrm{Au}$ is the binding center and it follows the order as $\mathrm{Au}>\mathrm{Cu}>\mathrm{Ag}$. The extent of covalent character in the $\mathrm{Ng}-\mathrm{M}$ bonds can be predicted from the WBI values. The WBI values corresponding to $\mathrm{Ng}-\mathrm{M}$ bonds also exhibit the similar trend having a lower value for lighter $\mathrm{Ng}$ atoms and a higher value for heavier analogues. The low value of WBI indicates the interaction as of noncovalent type which occurs in case of lighter $\mathrm{Ng}$ atoms and a large value of WBI indicates the significant covalent character in the Ng-M bond as in the cases of heavier 
analogues. In addition, for a given $\mathrm{Ng}$ and $\mathrm{Y}$ the $\mathrm{Au}$ analogues are most covalent followed by $\mathrm{Cu}$ and $\mathrm{Ag}$ analogues.

Electron density analysis provides further insight into the nature of bonding. Tables 3 and 4 represent different topological descriptors evaluated at the bond critical points (BCPs) of $\mathrm{Ng}-\mathrm{M}$ bonds present in these compounds. Local electron energy density $\left(\mathrm{H}\left(\mathbf{r}_{\mathbf{c}}\right)\right)$ is computed as the sum of local kinetic energy density $\left(\mathrm{G}\left(\mathbf{r}_{\mathbf{c}}\right)\right)$ and local potential energy density $\left(\mathrm{V}\left(\mathbf{r}_{\mathbf{c}}\right)\right)$. In general, the accumulation and depletion of electron density $\left(\rho\left(\mathbf{r}_{\mathbf{c}}\right)\right)$ reflecting from the corresponding negative and positive values of Laplacian of $\rho\left(\mathbf{r}_{\mathbf{c}}\right)\left(\nabla^{2} \rho\left(\mathbf{r}_{\mathbf{c}}\right)\right)$, respectively, at the BCPs indicate the covalent and noncovalent types of bonding, respectively. However, there are several systems involving $3 \mathrm{~d}$ transition metals and other heavier atoms, where a covalent bond cannot be explained using this descriptor. ${ }^{24,31}$

The $\mathrm{H}\left(\mathbf{r}_{\mathbf{c}}\right)$ is also used along with $\nabla^{2} \rho\left(\mathbf{r}_{\mathbf{c}}\right)$ to characterize a chemical bond. For example, if $\nabla^{2} \rho\left(\mathbf{r}_{\mathbf{c}}\right)>0$ and $\mathrm{H}\left(\mathbf{r}_{\mathbf{c}}\right)<0$ then the bond may be treated as partial
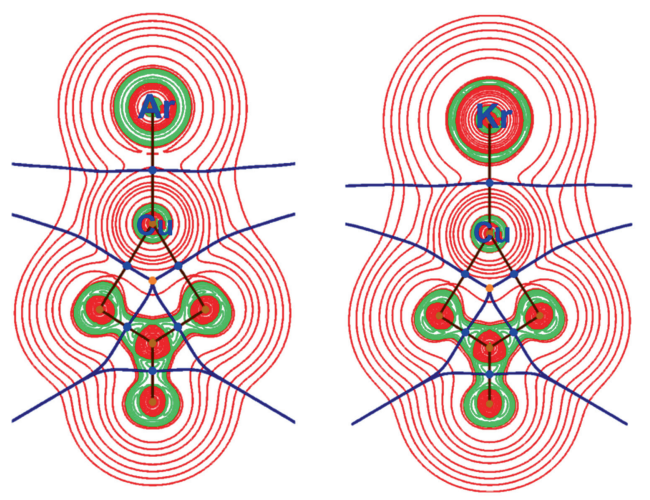

$\mathrm{ArCuNO}_{3}$
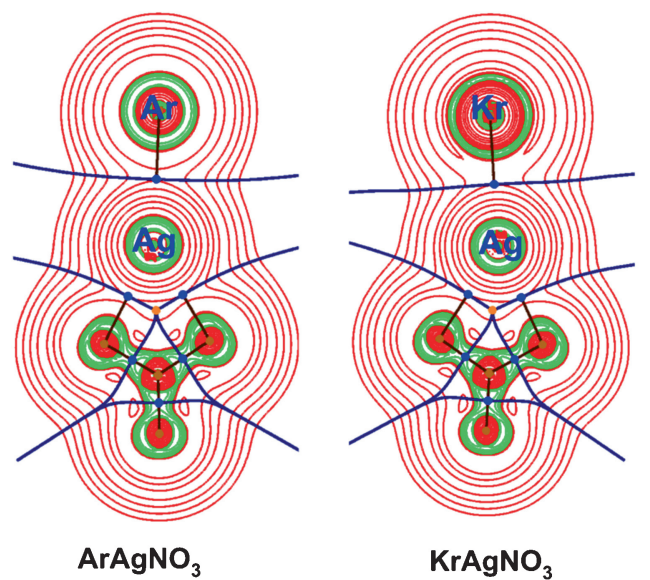

covalent in nature. ${ }^{32}$ Specifically when the bond has either zero or a very small bonding electron density $\left(\rho\left(\mathbf{r}_{\mathbf{c}}\right)\right)$ then it should be investigated with the aid of the $\mathrm{H}\left(\mathbf{r}_{\mathbf{c}}\right)$. In our cases, although the $\nabla^{2} \rho\left(\mathbf{r}_{\mathbf{c}}\right)$ is found to be positive at the BCPs of all $\mathrm{Ng}-\mathrm{Cu}, \mathrm{Ng}-\mathrm{Ag}$ and $\mathrm{Ng}$-Au bonds in all the systems under consideration, the bonding electron densities $\left(\rho\left(\mathbf{r}_{\mathbf{c}}\right)\right)$ are so small that we have to consider $\mathrm{H}\left(\mathbf{r}_{\mathbf{c}}\right)$. Almost all the $\mathrm{Ng}$ bound compounds contain negative $\mathrm{H}\left(\mathbf{r}_{\mathbf{c}}\right)$ value at the BCPs of $\mathrm{Ng}$ $\mathrm{M}$ bonds except for $\mathrm{ArAgNO}_{3}, \mathrm{ArAgSO}_{4}, \mathrm{Ar}_{2} \mathrm{Ag}_{2} \mathrm{SO}_{4}$ and $\mathrm{Ar}_{2} \mathrm{Ag}_{2} \mathrm{CO}_{3}$. It signifies that all these bonds are partially covalent in nature. It has to be mentioned here that $\mathrm{H}\left(\mathbf{r}_{\mathbf{c}}\right)$ becomes more negative in moving from $\mathrm{Ar}$ to heavier Ngs suggesting an increase in covalent character along the same. The contour plots of $\nabla^{2} \rho(\mathbf{r})$ are displayed in Figures 5 and 6 for $\mathrm{NgMNO}_{3}(\mathrm{M}=\mathrm{Cu}$, $\mathrm{Ag}$ ) and $\mathrm{NgMSO}_{4}(\mathrm{M}=\mathrm{Cu}, \mathrm{Ag})$, respectively (for the other compounds see Figures S2 in Supporting Information). In these plots, while the red-colored region displays the area having $\nabla^{2} \rho(\mathbf{r})>0$, the green-colored region shows the area of $\nabla^{2} \rho(\mathbf{r})<0$. It is obvious from
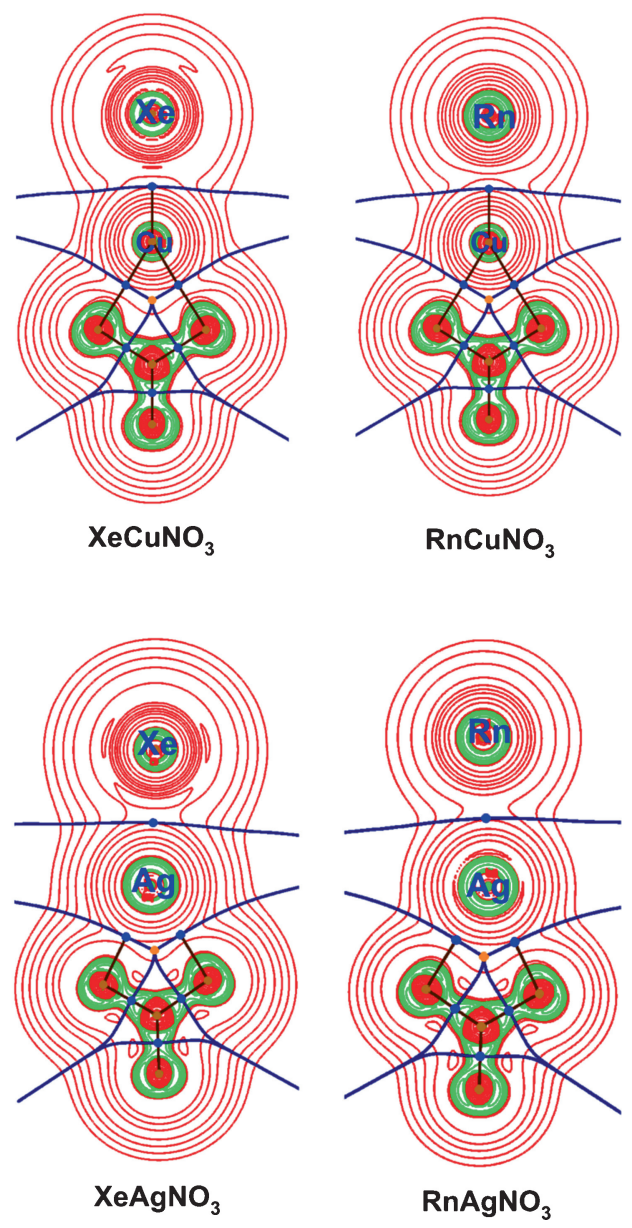

Figure 5. Contour plots of the Laplacian of the electron density of $\mathrm{NgMNO}_{3}(\mathrm{Ng}=\mathrm{Ar}, \mathrm{Kr}$, $\mathrm{Xe}, \mathrm{Rn}$ )at a particular plane computed at the MPW1B95/def2-TZVP level; the red-colored region shows the area of $\nabla^{2} \rho(\mathbf{r})>0$, whereas the green-colored region shows the area of $\left.\nabla^{2} \rho(\mathbf{r})<0\right)$. 

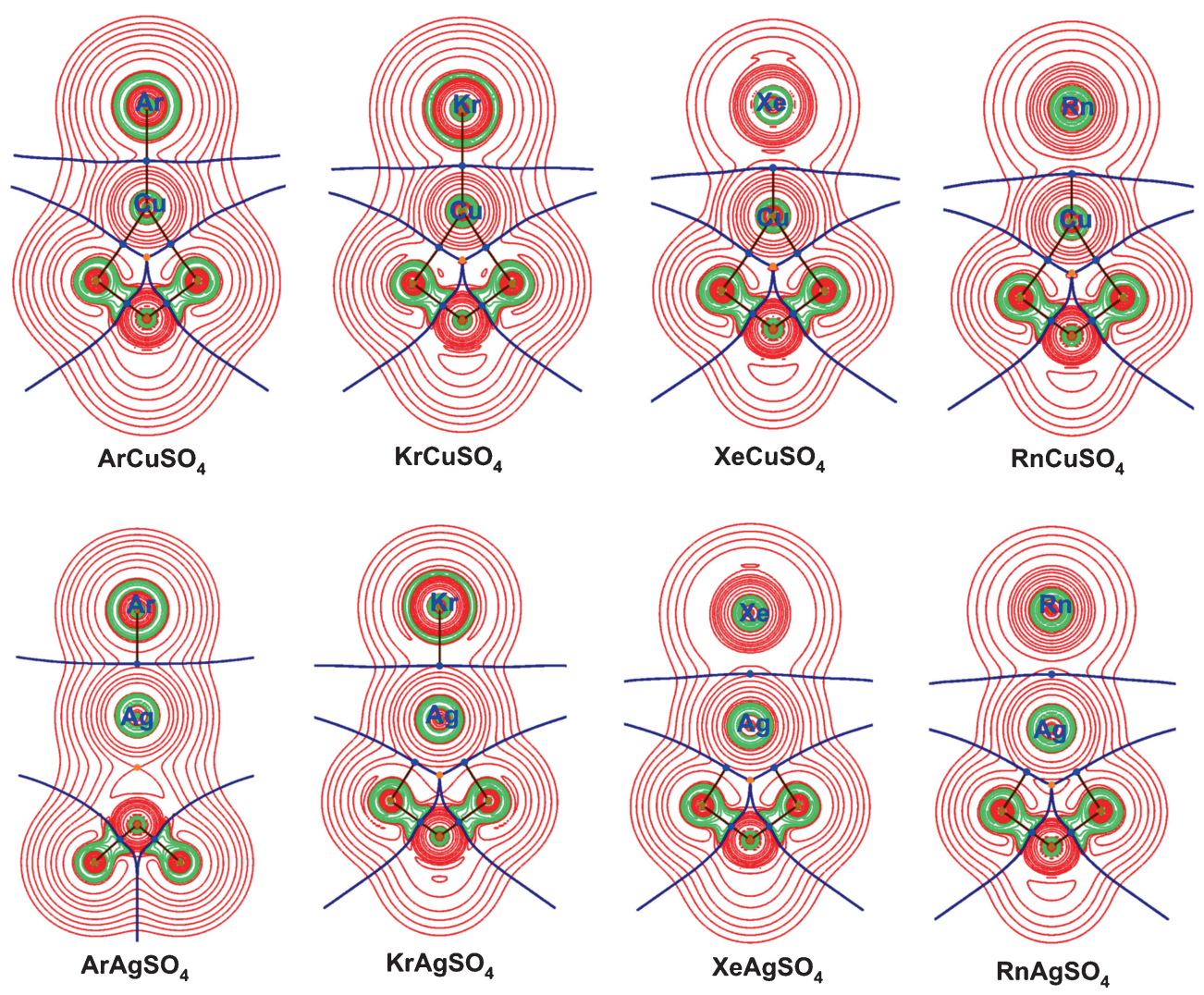

Figure 6. Contour plots of the Laplacian of the electron density of $\mathrm{NgMSO}_{4}(\mathrm{Ng}=\mathrm{Ar}, \mathrm{Kr}$, $\mathrm{Xe}, \mathrm{Rn}$ ) compounds at a particular plane computed at the MPW1B95/def2-TZVP level; the red-colored region shows the area of $\nabla^{2} \rho(\mathbf{r})>0$, whereas the green-colored region shows the area of $\left.\nabla^{2} \rho(\mathbf{r})<0\right)$.

the plots that there is no development of green region $\left(\nabla^{2} \rho(\mathbf{r})<0\right)$ in between $\mathrm{Ng}$ and $\mathrm{M}$. The shape of the valence orbitals of $\mathrm{Ng}$ atoms only gets deformed and the deformation continuously increases along Ar to Rn.

\section{Conclusions}

The Ng binding ability of $\mathrm{MY}$ and $\mathrm{M}_{2} \mathrm{Y}(\mathrm{M}=\mathrm{Cu}, \mathrm{Ag}$, $\mathrm{Au}$ and $\mathrm{Y}=\mathrm{NO}_{3}, \mathrm{SO}_{4}, \mathrm{CO}_{3}$ ) compounds is assessed in the present study. The $\mathrm{Ng}-\mathrm{M}$ bond dissociation energy $\left(\mathrm{D}_{0}\right)$ values for these metal nitrates, sulphates and carbonates vary within $5.1-13.2 \mathrm{kcal} / \mathrm{mol}, 2.3-$ $10.1 \mathrm{kcal} / \mathrm{mol}$ and $7.3-19.9 \mathrm{kcal} / \mathrm{mol}$ for $\mathrm{Cu}, \mathrm{Ag}$ and $\mathrm{Au}$ as binding sites, respectively. For a given $\mathrm{Ng}$ and $\mathrm{Y}, \mathrm{Au}$ analogue shows the highest $\mathrm{Ng}$ binding ability followed by $\mathrm{Cu}$ and $\mathrm{Ag}$. The $\mathrm{D}_{0}$ value also increases gradually from $\mathrm{Ar}$ to $\mathrm{Rn}$. Most of the dissociation processes are found to be endergonic in nature at room temperature, except for those involving $\mathrm{ArCuSO}_{4}, \mathrm{ArAgY}, \mathrm{KrAgY}$ $\left(\mathrm{Y}=\mathrm{NO}_{3}, \mathrm{SO}_{4}\right), \mathrm{Ar}_{2} \mathrm{Ag}_{2} \mathrm{Y}, \mathrm{Kr}_{2} \mathrm{Ag}_{2} \mathrm{Y}\left(\mathrm{Y}=\mathrm{CO}_{3}, \mathrm{SO}_{4},\right)$ and $\mathrm{Ar}_{2} \mathrm{Cu}_{2} \mathrm{SO}_{4}$. There is some degree of electron transfer from $\mathrm{Ng}$ to $\mathrm{M}$ center as highlighted by natural population analysis and it gradually increases in moving from Ar to its heavier congeners. The Wiberg bond indices indicate that there is partly covalent character in $\mathrm{Ng}-\mathrm{M}$ bonds, especially for $\mathrm{Xe}$ and $\mathrm{Rn}$ bound analogues. Increase in the HOMO-LUMO energy gap in the $\mathrm{Ng}$ bound analogues compared to that of the bare compounds reflects larger electronic stability of the former. The negative value of local electron energy density, $\mathrm{H}\left(\mathbf{r}_{\mathbf{c}}\right)$, at the bond critical points of $\mathrm{Ng}-\mathrm{M}$ bonds indicates the partial covalent character as provided by the electron density analysis.

\section{Supplementary Information (SI)}

Figures S1 and S2, and Table S1 are available as Supplementary Information at www.ias.ac.in/chemsci.

\section{Acknowledgments}

PKC thanks Department of Science \& Technology, New Delhi for the J. C. Bose National Fellowship. MG and SP thank Council of Scientific \& Industrial Research, New Delhi, for their fellowships. US would like to thank Centre for Theoretical studies (CTS), IIT Kharagpur for providing him CTS visiting fellowship. 


\section{References}

1. Bartlett N 1962 Proc. Chem. Soc. 218

2. Kossel W 1916 Ann. Phys. 49229

3. Pauling L 1932 J. Am. Chem. Soc. 543570

4. (a) Frenking G, Gauss W J and Cremer D 1988 J. Am. Chem. Soc. 110 8007; (b) Koch W, Liu B, Frenking G and 1990 J. Chem. Phys. 92 2464; (c) Frenking G, Koch W, Reichel F and Cremer D $1990 \mathrm{~J}$. Am. Chem. Soc. 112 4240; (d) Veldkamp A and Frenking G 1994 Chem. Phys. Lett. 226 11; (e) Jiménez-Halla C Ó, Fernández I and Frenking G 2009 Angew. Chem. Int. Ed. 48 366; (f) Mück L A, Timoshkin A Y, Hopffgarten M V and Frenking G 2009 J. Am. Chem. Soc. 131 3942; (g) Fernández I and Frenking G 2012 Phys. Chem. Chem. Phys. 14 14869; (h) Zhang Q, Chen M, Zhou M, Andrada D M and Frenking G 2015 J. Phys. Chem. A 1192543

5. (a) Pan S, Contreras M, Romero J, Reyes A, Chattaraj P K and Merino G 2013 Chem. Eur. J. 19 2322; (b) Pan S, Jalife S, Romero J, Reyes A, Merino G and Chattaraj P K 2013 Comput. Theor. Chem. 1021 62; (c) Pan S, Jalife S, Kumar R M, Subramanian V, Merino G and Chattaraj P K 2013 ChemPhysChem 14 2511; (d) Khatua M, Pan S and Chattaraj P K 2014 Chem. Phys. Lett. 610-611 351; (e) Pan S, Moreno D, Cabellos J L, Romero J, Reyes A, Merino G and Chattaraj P K 2014 J. Phys. Chem. A 118 487; (f) Khatua M, Pan S and Chattaraj P K 2014 J. Chem. Phys. 140 164306; (g) Pan S, Moreno D, Cabellos J L, Merino G and Chattaraj P K 2014 ChemPhysChem 15 2618; (h) Pan S, Moreno D, Merino G and Chattaraj P K 2014 ChemPhysChem 15 3554; (i) Pan S, Saha R and Chattaraj P K 2015 Int. J. Mol. Sci. 16 6402; (j) Saha R, Pan S and Chattaraj P K 2015 J. Phys. Chem. A 119 6746; (k) Pan S, Saha R, Kumar A, Gupta A, Merino G and Chattaraj P K 2016 Int. J. Quantum Chem. 116 1016; (1) Pan S, Saha R, Mandal S and Chattaraj P K 2016 Phys. Chem. Chem. Phys. 1811661

6. (a) Lundell J, Cohen A and Gerber R B 2002 J. Phys. Chem. A 106 11950; (b) Gerber R B 2005 Bull. Israel Chem. Soc. 18 7; (c) Khriachtchev L, Räsänen M and Gerber R B 2009 Acc. Chem. Res. 42 183; (d) Tsivion U and Gerber R B 2009 Chem. Phys. Lett. 482 30; (e) Feldman V I, Kobzarenko A V, Baranova I A, Danchenko A V, Sukhov F O, Tsivion E and Gerber R B 2009 J. Chem. Phys. 131151101

7. (a) Grochala W 2009 Pol. J. Chem. 83 87; (b) Lockyear J F, Douglas K, Price S D, Karwowska M, Fijałkowski K J, Grochala W, Remeš M, Roithová J and Schröder D 2010 J. Phys. Chem. Lett. 1 358; (c) Kurzydłowski D, Ejgierd-Zaleski P, Grochala W and Hoffmann R 2011 Inorg. Chem. 50 3832; (d) Grochala W 2012 Phys. Chem. Chem. Phys. 14 14860; (e) Szarek P and Grochala W 2015 J. Phys. Chem. A 1192483

8. (a) Antoniotti P, Bronzolino N and Grandinetti F 2003 J. Phys. Chem. A 107 2974; (b) Borocci S, Bronzolino N and Grandinetti F 2006 Chem. Eur. J. 12 5033; (c) Grandinetti F 2004 Int. J. Mass Spectrom. 237 243; (d) Borocci S, Bronzolino N and Grandinetti F 2005 Chem. Phys. Lett. 406 179; (e) Antoniotti P, Bottizzo E, Operti L, Rabezzana R, Borocc S and Grandinetti F $2010 \mathrm{~J}$.
Phys. Chem. Lett. 1 2006; (f) Operti L, Rabezzana R, Turco F, Borocci S, Giordani M and Grandinetti F 2011 Chem. Eur. J. 17 10682; (g) Borocci S, Giordani M and Grandinetti F 2015 J. Phys. Chem. A 1192383

9. (a) Jayasekharan T and Ghanty T K 2007 J. Chem. Phys. 127 114314; (b) Jayasekharan T and Ghanty T K 2008 J. Chem. Phys. 128 144314; (c) Jayasekharan T and Ghanty T K 2008 J. Chem. Phys. 129 184302; (d) Sirohiwal A, Manna D, Ghosh A, Jayasekharan T and Ghanty T K 2013 J. Phys. Chem. A 117 10772; (e) Manna D, Ghosh A and Ghanty T K 2013 J. Phys. Chem. A 117 14282; (f) Ghosh A, Manna D and Ghanty T K 2015 J. Phys. Chem. A 1192233

10. (a) Wijngaarden J V and Jäger W 2001 J. Chem. Phys. 115 6504; (b) Dham A K, McCourt F R and Dickinson A S 2007 J. Chem. Phys. 127 054302; (c) Han J, Philen D and Heaven M C 2006 J. Chem. Phys. 124054314

11. Claassen H H, Selig H and Malm J G 1962 J. Am. Chem. Soc. 843593

12. Hoppe R, Dähne W, Mattauch H and Rödder K 1962 Angew. Chem. Int. Ed. 1599

13. (a) Pettersson M, Lundell J and Räsänen M 1995 J. Chem. Phys. 103 205; (b) Pettersson M, Nieminen J, Khriachtchev L and Räsänen M 1997 J. Chem. Phys. 107 8423; (c) Pettersson M, Lundell J, Isamieni L and Räsänen M 1998 J. Am. Chem. Soc. 120 7979; (d) Pettersson M, Lundell J, Khriachtchev L and Räsänen M 1998 J. Chem. Phys. 109 618; (e) Pettersson M, Khriachtchev L, Lundell J and Räsänen M 1999 J. Am. Chem. Soc. 121 11904; (f) Khriachtchev L, Pettersson M, Runeberg N, Lundell J and Räsänen M 2000 Nature 406 874; (g) Khriachtchev L, Pettersson M, Lignell A and Räsänen M 2001 J. Am. Chem. Soc. 123 8610; (h) Khriachtchev L, Pettersson M, Lundell J, Tanskanen H, Kiviniemi T, Runeberg N and Räsänen M 2003 J. Am. Chem. Soc. 125 1454; (i) Khriachtchev L, Tanskanen H, Cohen A, Gerber R B, Lundell J, Pettersson M, Kiljunen H and Räsänen M 2003 J. Am. Chem. Soc. 125 6876; (j) Tanskanen H, Khriachtchev L, Lundell J, Kiljunen H and Räsänen M 2003 J. Am. Chem. Soc. 12516361

14. (a) Feldman V I and Sukhov F F 1996 Chem. Phys. Lett. 225 425; (b) Feldman V I, Sukhov F F and Orlov A Y 1997 Chem. Phys. Lett. 280 507; (c) Khriachtchev L, Tanskanen H, Pettersson M, Rasanen M, Ahokas J, Kunttu H and Feldman V 2002 J. Chem. Phys. 1165649 ; (d) Feldman V I, Sukhov F F, Orlov A Yu and Tyulpina I V 2003 J. Am. Chem. Soc. 125 4698; (e) Feldman V I, Kobzarenko A V, Baranova I A, Danchenko A V, Sukhov F F, Tsivion E and Gerber R B 2009 J. Chem. Phys. 131 151101; (f) Ryazantsev S V, Kobzarenko A V and Feldman V I 2013 J. Chem. Phys. 139124315

15. (a) Thompson C A and Andrews L 1994 J. Am. Chem. Soc. 116 423; (b) Thompson C A and Andrews L 1994 J. Chem. Phys. 100 8689; (c) Wang X, Andrews L, Willmann K, Brosi F and Riedel S 2012 Angew. Chem. Int. Ed. 51 10628; (d) Li J, Bursten B E, Liang B and Andrews L 2002 Science 295 2242; (e) Liang B, Andrews L, Li J and Bursten B E 2002 J. Am. Chem. Soc. 124 9016; (f) Wang X, Andrews L, Li J and Bursten B E 2004 Angew. Chem. Int. Ed. 432554

16. (a) Evans C J, Lesarri A and Gerry M C L $2000 \mathrm{~J}$. Am. Chem. Soc. 122 6100; (b) Evans C J and Gerry M C L 2000 J. Chem. Phys. 112 1321; (c) Evans C J and Gerry 
M C L 2000 J. Chem. Phys. 112 9363; (d) Evans C J, Rubinoff D S and Gerry M C L 2000 Phys. Chem. Chem. Phys. 2 3943; (e) Reynard L M, Evans C J and Gerry M C L 2001 J. Mol. Spectrosc. 206 33; (f) Walker N R, Reynard L M and Gerry M C L 2002 J. Mol. Struct. 612 109; (g) Michaud J M, Cooke S A and Gerry M C L 2004 Inorg. Chem. 43 3871; (h) Thomas J M, Walker N R, Cooke S A and Gerry M C L 2004 J. Am. Chem. Soc. 126 1235; (i) Cooke S A and Gerry M C L 2004 Phys. Chem. Chem. Phys. 6 3248; (j) Cooke S A and Gerry M C L 2004 J. Am. Chem. Soc. 126 17000; (k) Michaud J M and Gerry M C L 2006 J. Am. Chem. Soc. 1287613

17. (a) Emara A A and Schrobilgen G J 1992 Inorg. Chem. 31 1323; (b) Schumacher G A and Schrobilgen G J 1983 Inorg. Chem. 22 2178; (c) Smith G L, Mercier H P and Schrobilgen G J 2007 Inorg. Chem. 46 1369; (d) Schrobilgen G J 1988 J. Chem. Soc., Chem. Commun. 13 863; (e) Hughes M, Brock D S, Mercier H P A and Schrobilgen G J 2011 J. Fluorine Chem. 132 660; (f) Smith G L, Mercier H P A and Schrobilgen G J 2011 Inorg. Chem. 49 12359; (g) Brock D S, Mercier H P A and Schrobilgen G J 2013 J. Am. Chem. Soc. 135 5089; (h) Debackere J R, Mercier H P A and Schrobilgen G J 2014 J. Am. Chem. Soc. 1363888

18. (a) Pyykkö P 1995 J. Am. Chem. Soc. 117 2067; (b) Schröder D, Schwarz H, Hrušák J and Pyykkö P 1998 Inorg. Chem. 37624

19. Seidel S and Seppelt K 2000 Science 290117

20. Wang X, Andrews L, Brosi F and Riedel S 2013 Chem. Eur. J. 191397

21. (a) Zhang P-X, Zhao Y-F, Hao F-Y, Zhang G-H, Song X-D and Li X-Y 2008 Mol. Phys. 106 1007; (b) Zhang P X, Zhao Y F, Hao F Y and Li X Y 2008 Int. J. Quantum Chem. 108937

22. Pan S, Gupta A, Saha R, Merino G and Chattaraj P K 2015 J. Comp. Chem. 3629

23. (a) Ghanty T K 2005 J. Chem. Phys. 123 074323; (b) Ghanty T K 2006 J. Chem. Phys. 124124304
24. Bader R F W 1990 In Atoms in Molecules: A Quantum Theory (Oxford, UK: Clarendon Press) pp. 312314

25. Lai T-Y, Yang C-Y, Lin H-J, Yang C-Y and Hu W-P 2011 J. Chem. Phys. 134244110

26. Peterson K A, Figgen D, Goll E, Stoll H and Dolg M 2003 J. Chem. Phys. 11911113

27. Wiberg K B 1968 Tetrahedron 241083

28. Gaussian 09, Revision C 1, Frisch M J et al. 2009 Gaussian, Inc., Wallingford CT

29. Lu T and Chen F W 2012 J. Comput. Chem. 33580

30. (a) Pearson R G 1987 J. Chem. Educ. 64 561; (b) Parr R G and Chattaraj P K $1991 \mathrm{~J}$. Am. Chem. Soc. 113 1854; (c) Chattaraj P K and Parr R G 1993 Density functional theory of chemical hardness In Chemical Hardness (Structure and Bonding) Vol. $80 \mathrm{~K}$ D Sen and D M P Mingos (Eds.) (Berlin: Springer- Verlag) pp. 11-25; (d) Pan S, Sola M and Chattaraj P K 2013 J. Phys. Chem. A 1171843

31. (a) Cioslowski J and Mixon S T 1992 Can. J. Chem. 70 443; (b) Cioslowski J and Mixon S T 1992 J. Am. Chem. Soc. 114 4382; (c) Haaland A, Shorokhov D J and Tverdova N V 2004 Chem. Eur. J. 10 4416; (d) Krapp A and Frenking G 2007 Chem. Eur. J. 13 8256; (e) Poater J, Visser R, Solà M and Bickelhaupt F M 2007 J. Org. Chem. 72 1134; (f) Cerpa E, Krapp A, Vela A and Merino G 2008 Chem. Eur. J. 14 10232; (g) Cerpa E, Krapp A, Flores-Moreno R, Donald K J and Merino G 2009 Chem. Eur. J. 15 1985; (h) Macchi P, Proserpio D M and Sironi A J 1998 J. Am. Chem. Soc. 120 13429; (i) Macchi P, Garlaschelli L, Martinengo S and Sironi A J 1999 J. Am. Chem. Soc. 121 10428; (j) Novozhilova I V, Volkov A V and Coppens P J 2003 J. Am. Chem. Soc. 125 1079; (k) Pan S, Gupta A, Mandal S, Moreno D, Merino G and Chattaraj P K 2015 Phys. Chem. Chem. Phys. 17972

32. Cremer D and Kraka E 1984 Angew. Chem. Int. Ed. Engl. 23627 